\title{
How to Perform Cataract Surgery on Patients Implanted with Phakic IOL
}

\section{Laurent Khaitrine*}

Department of Ophthalmology, Rue De Courlancy, France

*Corresponding author: Laurent Khaitrine, Department of Ophthalmology, Rue De Courlancy, France.

Received Date: February 03, 2020

Published Date: February 13, 2020

\section{Introduction}

Refractive corneal surgery (Lasik) is not considered as the best option to correct high myopia ( $>10 \mathrm{~d}$ )

- When photo-ablation is too deep, stromal residual bed gets too thin, thus creating a great risk for complications such as postoperative corneal ectasia

- $\quad$ Photo-ablation depth can be decreased by narrowing the optical zone but then postoperative visual quality tends to be poor, especially in photopic conditions

To prevent these postoperative complications, using a phakic IOL (iris claw) either in front or behind the iris appears to be the best option to correct high myopia for young patients (note: cataract surgery can be considered for older ones).

A few years after the phakic IOL implantation, crystalline lens surgery is typically required for two main reasons:

- A decrease in total number of endothelial cells that has to be regularly monitored by specular microscopy

- The early appearance of cataract, given that patients with high myopia tend to develop cataract earlier than non-myopic ones

This second case raises the question of how to perform cataract surgery and remove the iris claw has at the same time. Three surgical techniques can be considered:

- $\quad$ Remove the iris claw before a regular cataract surgery

- $\quad$ Remove the iris claw after a regular cataract surgery

- $\quad$ Remove the iris claw after the cataract surgery using Femto Laser for incision, capsulorhexis and fragmentation.

Remove the Iris Claw Before a Regular Cataract Surgery

This first technique consists of removing the iris claw at the beginning of the surgery through a large incision (given the iris claw width of 5-6 mm diameter). Phacoemulsification has then to be performed through a narrower incision to prevent the anterior chamber to be very shallow and maneuvers to get more complicated. Therefore, sutures have to be put to narrow the incision before starting the regular cataract. Overall, the surgery remains difficult due to leaks through the cornea (Figure 1).

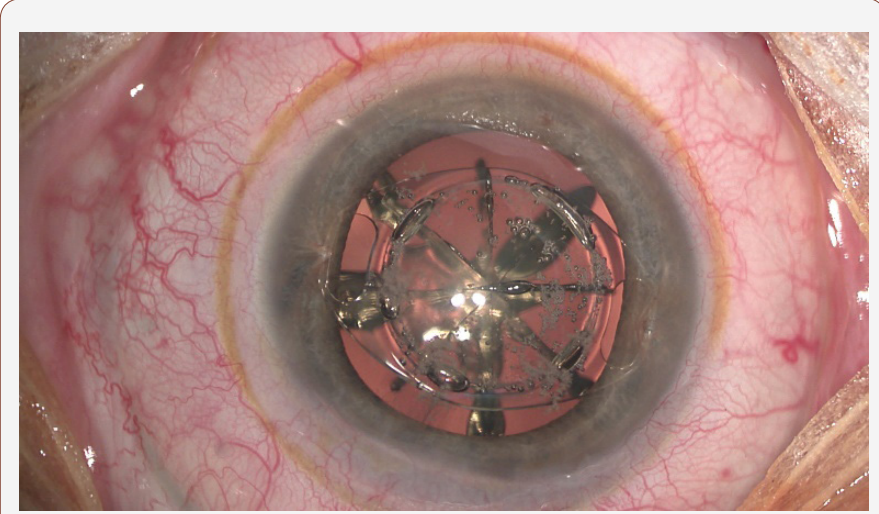

Figure 1

Remove the Iris Claw After a Regular Cataract Surgery

This second technique is to perform phacoemulsification with a classic $2 \mathrm{~mm}$ incision in a "normal way" through or behind the phakic IOL and to replace it at the end of the surgery. No specific problem stands out when performing capsulorhexis or phacoemulsification behind the phakic IOL, but it is very important to use viscoelastic several times during the surgery to protect the endothelium cells. At the end the cataract surgery, the pupil is constricted and the incision is enlarged to remove the iris claw (Figure 2) 


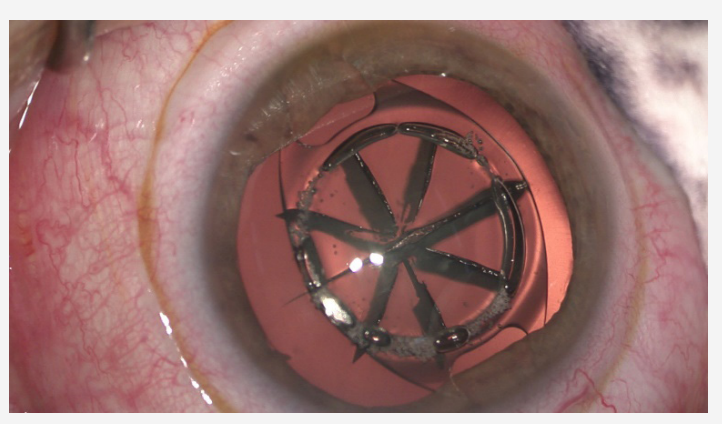

Figure 2

Remove the Iris Claw After the Cataract Surgery

\section{Using Femto Laser}

This third technique is the one we've been using for several years with the Femto Laser (except if the pupil is too narrow or for deep set eyes).

- $\quad$ Femto Laser is used at the beginning of the surgery to perform main \& port side incisions, capsulorhexis and fragmentation. Then the anterior capsule and the six or eight quarters of the lens are removed. Finally, pseudophakiciol is placed in a bag, pupil is constricted, incision is enlarged and iris claw is removed.

- This technique raises the question of whether the beam of the Femto Laser for cataract surgery can easily go through the phakic IOL? The answer so far has been positive and results have been ever more accurate than by using the two techniques mentioned above.

- $\quad$ Several benefits of this method stand out:

a. The incision in three planes is perfect

b. The capsulorhexis is circular, perfectly round for an exact centering of the phakic IOL

c. The pre-fragmentation using the Femto Laser brings a clear advantage to better protect corneal endothelial cells: prefragmentation of the lens produces less ultrasound with the phaco machine, which leads to less energy dissipated and thus better preservation of the corneal endothelial cells. This advantage also applies to fuchs dystrophy.

Overall, using Femto Laser to perform cataract surgery on patients implanted with Phakic IOL lenses leads to better results than historical surgery techniques.

\section{Acknowledgement}

None.

\section{Conflicts of Interest}

No conflict of interest. 